\title{
Antenatal Pertussis Vaccination: are we implementing best evidence into practice?
}

Sushena KRISHNASWAMY1, 2, Euan Wallace ${ }^{1,3}$, 4, Jim BUTTERY³, 5, 6, 7, 8, Michelle GILES 1

${ }^{1}$ Department of Obstetrics and Gynaecology, Monash University, Clayton, Victoria, Australia

${ }^{2}$ Department of Infectious Diseases, Monash Health, Clayton, Victoria, Australia

${ }^{3}$ The Ritchie Centre, Hudson Institute, Clayton, Victoria, Australia

${ }^{4}$ Department of Obstetrics, Monash Health, Melbourne, Australia

${ }^{5}$ Infection and Immunity, Monash Children's Hospital, Clayton, Victoria, Australia

${ }^{6}$ Monash Immunisation, Monash Health, Clayton, Victoria, Australia

${ }^{7}$ Department of Paediatrics, Monash University, Clayton, Victoria, Australia

${ }^{8}$ SAEFVIC, Murdoch Childrens Research Institute, Parkville, Victoria, Australia

Corresponding Author: Dr. Sushena Krishnaswamy, Monash Infectious Diseases, Monash Health, 246 Clayton Road, Clayton 3168, Australia. Tel: (03) 95944564.

Email: sushena.krishnaswamy@monashhealth.org

\section{Disclosures}

Dr. Krishnaswamy receives funding from a Monash University Australian Postgraduate Association Scholarship, Victorian Department of Health and Ageing, and a Small Project Grant from Glaxo Smith Kline for her PhD on antenatal pertussis vaccination

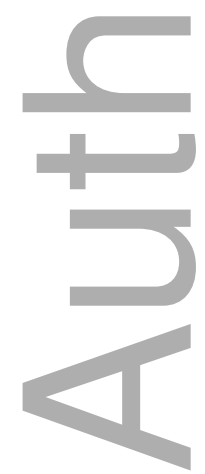

This is the author manuscript accepted for publication and has undergone full peer review but has not been through the copyediting, typesetting, pagination and proofreading process, which may lead to differences between this version and the Version of Record. Please cite this article as doi: 10.1111/ajo.12554

This article is protected by copyright. All rights reserved 
Received Date : 13-Jun-2016

Revised Date : 22-Aug-2016

Accepted Date : 06-Sep-2016

Article type : Review

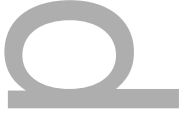

Antenatal Pertussis Vaccination: are we implementing best evidence into practice?

Word count (abstract): 71

Word Count (main text): 1570

Keywords: pertussis; maternal immunisation; vaccine acceptance; vaccines in pregnancy; vaccine attitudes

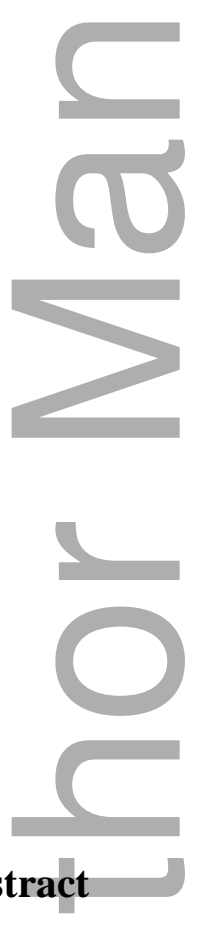

Maternal immunisation is the most effective strategy to reduce infant morbidity and mortality from pertussis infection, and is now standard of care in many countries, including Australia. However uptake cannot be guaranteed unless the barriers to implementing programs locally are understood. Education and resources for antenatal care providers, embedding vaccination within antenatal care, and provision of culturally appropriate information for pregnant women are integral to a successful antenatal vaccination program.

This article is protected by copyright. All rights reserved 


\section{Introduction}

Pertussis infection causes significant morbidity and mortality, particularly in children less than six months of age before they have received the protective immunological benefit of at least two doses of pertussis containing vaccine. Up to two thirds of infected babies require hospitalisation, and nearly 1 in 100 under 6 months of age die. ${ }^{1}$ Sadly, for over two decades Australia has had the highest reported rates of pertussis in the world. ${ }^{2}$

Multiple approaches to reducing risk to newborns have been explored. Vaccination reduces the incidence of infection more than 20-fold, but at least two doses are required to confer protection. "1,2 "Cocooning" was implemented in 2011-12 to reduce the 50-60\% of infant infections acquired from parents. But achieving a complete cocoon is costly, resource and time-intensive, and logistically challenging to implement. ${ }^{3,4}$

The Australian Immunisation Handbook was updated in March 2015 to recommend pertussis vaccination to pregnant women in the third trimester of every pregnancy regardless of prior vaccination history. ${ }^{1}$ This was endorsed by the Royal Australian and New Zealand College of Obstetricians and Gynaecologists. All Australian States and Territories have funded maternal vaccination programs since June 2015, initiatives that have seen vaccination uptake increase from $8 \%$ to $72 \% .^{5}$

This article reviews the evidence for maternal vaccination, identifies barriers to uptake, and highlights areas for future research. A literature search was performed using key words and restricting manuscripts to English.

\section{Antenatal Vaccination}

Antenatal pertussis vaccination achieves two for one protection: of the pregnant woman who may be susceptible due to waning immunity, and of her baby through placental transfer of maternal antibodies.

\section{Placental transfer of maternal antibodies}


Active and passive transfer of maternal IgG across the placenta provides transient passive immunity to the newborn with fetal antibody titres rising two weeks after maternal vaccination. However, most pregnant women do not have adequate levels of anti-pertussis IgG for placental transfer to be effective and therefore require a booster dose during pregnancy. ${ }^{6}$

\section{Does maternal vaccination reduce newborn pertussis infection?}

Two major studies have reported the efficacy of maternal vaccination in preventing laboratory-confirmed pertussis in infants under three months of age to be $91-93 \%$. $^{7,8}$

\section{What is the best time for maternal pertussis vaccination?}

Longer latency between maternal vaccination and delivery may facilitate greater antibody transfer. Third trimester vaccination is effective but recent studies report significantly higher maternal and infant antibody levels with second trimester vaccination than with third. ${ }^{3,6,9-11}$ Vaccination prior to 20 weeks however leads to waning antibody levels before infant vaccination at 6 weeks of age. ${ }^{6}$ Other benefits of second trimester vaccination include protection for the $7 \%$ of babies born preterm who might have inadequate levels if vaccinated in third trimester, and a wider "immunisation window" to aid implementation.

\section{Are there concerns about maternal pertussis vaccination?}

\section{Blunting of infant immune responses}

Based on studies of live vaccines (measles and oral polio) but also whole cell pertussis vaccine, high levels of maternally-derived antibodies in the newborn may blunt the infant's own immune response to primary immunisation. ${ }^{12}$

Three recent studies reported lower antibody levels after a primary series of acellular pertussis-containing vaccines in infants of mothers vaccinated antenatally. However, there was no difference following the booster dose at 18months of age (a dose that has been recently reintroduced in Australia), suggesting memory response may be unaffected. ${ }^{3,9,13}$ 
Reassuringly, this blunted response does not appear to be clinically significant, as there is no evidence of increasing pertussis infections in late infancy in the UK where there has been high antenatal immunisation coverage since 2012.

\section{$\underline{\text { Is antenatal pertussis vaccination safe? }}$}

More than half a million pregnant women have received the vaccine. Cohort data from over 40000 pregnant women identified no adverse birth outcomes or increase in medically attended adverse events following vaccination. ${ }^{14,15}$ Using short message service (sms) surveillance following vaccination, 1 in 10 antenatally vaccinated women in Western Australia reported an adverse reaction within a week of vaccination, predominantly local reactions, particularly if previous pertussis vaccination was recent ( $11 \%$ vs $6 \%$ ) with few seeking medical attention for the same. $^{16}$

\section{Is repeated tetanus vaccination problematic?}

Given guidelines recommend a pertussis-containing vaccine in every pregnancy, concerns have been raised about women having repeated tetanus vaccination. Linked vaccination and outcome data from 30000 previously tetanus-vaccinated women who were subsequently vaccinated in pregnancy observed no difference in the rate of medically-attended adverse events or adverse birth outcomes. ${ }^{17}$

\section{Current recommendations and implementation Recommendations}

In 2015 the Global Pertussis Initiative recommended prioritisation of maternal vaccination to decrease pertussis-related infant mortality. Despite such recommendations, uptake has been variable, rapidly achieving $60 \%$ in the $\mathrm{UK}^{11}$ but as low as $14 \%$ in some states in the US. ${ }^{4}$ Even within Australia in the FluMUM study uptake varied from $26 \%$ to $62 \%$ in $2015 .^{5}$ Understanding the factors contributing to such variability is central to implementing a successful vaccination program.

\section{$\underline{\text { Systems-related factors }}$}


Antenatal care is provided by a range of healthcare providers (HCP) including general practitioners (GPs), obstetricians and midwives. Inevitably, this leads to uncertainty about whose role it is to discuss, recommend and provide vaccination. Traditionally obstetricians' expertise has been high risk rather than routine pregnancy care and midwives have been educators and not required to administer vaccinations. GPs are well equipped to immunise out of hospital settings but do not have the same facilities to do so in hospital antenatal clinics. Delineation of each HCP's role would provide clarity for HCP and women and ensure vaccination is not overlooked.

Knowledge and development of resources to support HCP is important. While researchers and policy makers may reference the Australian Immunisation Handbook, antenatal HCP who have not been familiar with immunisation until recently, may consult the hospital intranet or local guidelines. Provision of consistent, up to date, and easily accessible evidence-based guidelines to HCP should be ensured.

Programs are more likely to be successful if vaccination becomes embedded in routine pregnancy care. ${ }^{18}$ While currently not available in many pregnancy care settings, infrastructure to maintain and monitor cold chain and staff trained in immunisation are vital. Additionally, changes such as inclusion of antenatal pertussis in the pregnancy record would serve as a prompt for discussion and administration of vaccine and aid evaluation of safety and effectiveness. Currently only the antenatal record in Queensland includes such reminders for pertussis. ${ }^{18}$

\section{Healthcare provider factors}

HCPs have differing knowledge, experience and comfort with discussing and providing vaccination to pregnant women. Vaccination being a relatively recent development, many antenatal HCP do not traditionally see vaccination as within their remit. This needs a significant cultural shift for any vaccine program to be successful.

Midwives are frequently the first HCP a pregnant woman consults and are thereby well placed to advise women. A survey of midwives attitudes to post-partum pertussis vaccination found midwives were less confident than hospital nurses and immunisation providers in providing vaccine advice, and were more likely to administer vaccine if they believed immunisation to be part of their job and could 
easily integrate it into their workload. ${ }^{19}$ Researching attitudes to antenatal vaccination and equipping midwives with the necessary knowledge and support will be integral.

Obstetricians are significantly more likely to recommend vaccination if they have knowledge of national recommendations (OR 23.33), routinely recommend influenza vaccine (OR 12.5), are able to administer vaccines in their rooms (OR 7.01), and receive influenza vaccine themselves (OR 8.36). ${ }^{4}$

\section{Consumer factors}

The most common reasons pregnant women decline vaccination are lack of recommendation by HCPs, safety concerns, and lack of awareness of vaccine recommendations. ${ }^{18,20}$ Women are more inclined to have pertussis vaccination than influenza as they perceive pertussis as for the benefit of their baby compared to influenza vaccine which they perceive as solely for their own benefit. ${ }^{20}$ Women look to their HCP to provide evidence-based information and provision of such would overcome a number of these incorrect assumptions.

Women from culturally and linguistically diverse backgrounds and Aboriginal and Torres Strait Islanders (ATSI) have been underrepresented in research to date. In the FluMUM study only $34 \%$ of ATSI women were vaccinated against pertussis compared to $43 \%$ overall. ${ }^{5}$ Achieving universal vaccination engenders us to examine barriers and uptake in all groups particularly those traditionally known to underutilise maternal services.

The impact of the media as a driver of vaccination cannot be underestimated. ${ }^{20}$ Social media, with sites such as the Light for Riley Facebook page, has proven to be a powerful tool in disseminating personal stories in a much more accessible way than traditional information sources. Social media may play an even more important role in reaching groups with less healthcare engagement.

\section{Lessons Learned}

- Antenatal care provider recommendation is the strongest driver of vaccination 
- Systemic changes to embed vaccination into routine pregnancy care are vital

- The role of social media and personal stories in influencing consumer attitudes cannot be underestimated

\section{Priorities for Future Research}

- Studies on antenatal care provider and consumer barriers to uptake in the Australian context are needed

- Differences/ needs of specific cultural groups and ATSI must be examined to achieve universal vaccination

\section{Summary}

Maternal immunisation has emerged as the most effective strategy to reduce the morbidity and mortality of newborn pertussis infection. While uptake has been more enthusiastic than for maternal influenza vaccine, challenges to implementation in Australia remain. It is imperative to incorporate vaccination into routine antenatal care, identify the unique barriers to uptake in Australia, particularly in culturally and linguistically diverse and Aboriginal and Torres Strait Islander women. Ongoing surveillance to rapidly detect any unexpected adverse outcomes at a population level, will also be key to the success of current and future antenatal vaccination programs.

\section{References}

1. Australian Technical Advisory Group on Immunisation (ATAGI). The Australian immunisation handbook 10th ed (2015 update). Canberra: Australian Government Department of Health; 2015.

2. McIntyre PB, Nolan TM. Pertussis control: where to now? Med J Aust. 2014;200(6):306-7.

3. Hardy-Fairbanks AJ, Pan SJ, Decker MD, Johnson DR, Greenberg DP, Kirkland $\mathrm{KB}$, et al. Immune responses in infants whose mothers received Tdap vaccine during pregnancy. Pediatr Infect Dis J. 2013;32(11):1257-60.

4. Bonville CA, Cibula DA, Domachowske JB, Suryadevara M. Vaccine attitudes and practices among obstetric providers in New York State following the recommendation 
for pertussis vaccination during pregnancy. Hum Vaccin Immunother. 2015;11(3):713-8.

5. Andrews R, editor Maternal Immunisation in Australia- how are we going? SA Vaccinology Update Conference; 2015; National Wine Centre- Adelaide, Australia. 6. Healy CM, Rench MA, Baker CJ. Importance of timing of maternal combined tetanus, diphtheria, and acellular pertussis (Tdap) immunization and protection of young infants. Clin Infect Dis. 2013;56(4):539-44.

7. Amirthalingam G, Andrews N, Campbell H, Ribeiro S, Kara E, Donegan K, et al. Effectiveness of maternal pertussis vaccination in England: an observational study. The Lancet. 2014;384(9953):1521-8.

8. Dabrera G, Amirthalingam G, Andrews N, Campbell H, Ribeiro S, Kara E, et al. A case-control study to estimate the effectiveness of maternal pertussis vaccination in protecting newborn infants in England and Wales, 2012-2013. Clin Infect Dis.

2015;60(3):333-7.

9. Munoz FM, Bond NH, Maccato M, Pinell P, Hammill HA, Swamy GK, et al. Safety and immunogenicity of tetanus diphtheria and acellular pertussis (Tdap) immunization during pregnancy in mothers and infants: a randomized clinical trial. JAMA. 2014;311(17):1760-9.

10. Naidu M, Muljadi R, Davies-Tuck M, Wallace E, Giles M. The optimal gestation for pertussis vaccination during pregnancy - A prospective cohort study, . Am J Obstet Gynecol. 2016.

11. Eberhardt C, Blanchard- Rohner G, Lemaitre B, Boukrid M, Combescure C, Othenin-Girard V, et al. Maternal immunization earlier in pregnancy maximizes antibody transfer and expected infant seropositivity against pertussis. Clin Infect Dis. 2016.

12. Jones C, Pollock L, Barnett SM, Battersby A, Kampmann B. The relationship between concentration of specific antibody at birth and subsequent response to primary immunization. Vaccine. 2014;32(8):996-1002.

13. Ladhani SN, Andrews NJ, Southern J, Jones CE, Amirthalingam G, Waight PA, et al. Antibody Responses After Primary Immunization in Infants Born to Women Receiving a Pertussis-containing Vaccine During Pregnancy: Single Arm Observational Study With a Historical Comparator. Clin Infect Dis. 2015;61(11):1637-44. 
14. Donegan K, King B, Bryan P. Safety of pertussis vaccination in pregnant women in UK: observational study. BMJ. 2014;349:g4219.

15. Kharbanda EO, Vazquez-Benitez G, Lipkind HS, Klein NP, Cheetham TC, Naleway A, et al. Evaluation of the association of maternal pertussis vaccination with obstetric events and birth outcomes. JAMA. 2014;312(18):1897-904.

16. Regan AK, Tracey LE, Blyth CC, Richmond PC, Effler PV. A prospective cohort study assessing the reactogenicity of pertussis and influenza vaccines administered during pregnancy. Vaccine. 2016;34:2299-304.

17. Sukumaran L, McCarthy NL, Kharbanda EO, McNeil MM, Naleway AL, Klein NP, et al. Association of Tdap Vaccination With Acute Events and Adverse Birth Outcomes Among Pregnant Women With Prior Tetanus-Containing Immunizations. JAMA. 2015;314(15):1581-7.

18. Webb H, Street J, Marshall H. Incorporating immunizations into routine obstetric care to facilitate Health Care Practitioners in implementing maternal immunization recommendations. Hum Vaccin Immunother. 2014;10(4):1114-21.

19. Robbins SC, Leask J, Hayles EH, Sinn JK. Midwife attitudes: an important determinant of maternal postpartum pertussis booster vaccination. Vaccine.

2011;29(34):5591-4.

20. Wiley KE, Cooper SC, Wood N, Leask J. Understanding Pregnant Women's Attitudes and Behavior Toward Influenza and Pertussis Vaccination. Qual Health Res. 2015;25(3):360-70.

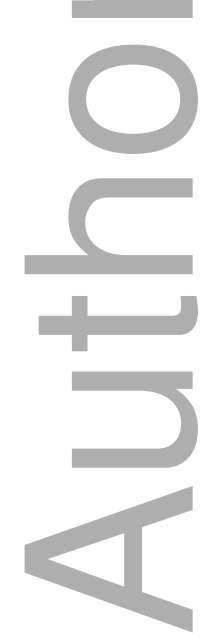




\section{University Library}

\section{- M M N E R VA A gateway to Melbourne's research publications}

Minerva Access is the Institutional Repository of The University of Melbourne

Author/s:

Krishnaswamy, S;Wallace, E;Buttery, J;Giles, M

Title:

Antenatal pertussis vaccination: Are we implementing best evidence into practice?

Date:

2016-12-01

Citation:

Krishnaswamy, S., Wallace, E., Buttery, J. \& Giles, M. (2016). Antenatal pertussis vaccination: Are we implementing best evidence into practice?. AUSTRALIAN \& NEW ZEALAND JOURNAL OF OBSTETRICS \& GYNAECOLOGY, 56 (6), pp.552-555. https:// doi.org/10.1111/ajo.12554.

Persistent Link:

http://hdl.handle.net/11343/291821 